\section{SCIENCE CHINA \\ Physics, Mechanics \& Astronomy}

\title{
Photoelectron holography for tunneling ionization
}

\author{
XueBin Bian* \\ State Key Laboratory of Magnetic Resonances and Atomic and Molecular Physics, Wuhan Institute of Physics and Mathematics, Innovation \\ Academy for Precision Measurement Science and Technology, Chinese Academy of Sciences, Wuhan 430071, China
}

Received May 2, 2021; accepted June 1, 2021; published online June 25, 2021

Citation: X. B. Bian, Photoelectron holography for tunneling ionization, Sci. China-Phys. Mech. Astron. 64, 283031 (2021), https://doi.org/10.1007/s11433021-1728-6

Holography was invented by Gabor [1]. It records the interference between the reference and signal waves. Both the amplitude and phase information could be obtained. It has been widely used for imaging objects. Laser-induced photoelectron holography $(\mathrm{PH})$ was proposed by Spanner et al. [2]. $\mathrm{PH}$ is based on the interference between the directly ionized electrons (reference) and rescattered electrons (signal) from the same target. Huismans et al. [3] realized the experimental study by using excited Xe with a $7 \mu \mathrm{m}$ freeelectron laser. The dynamics of the direct electrons are known by the strong-field approximation method. The rescattered electrons carry novel information of the atomic and molecular structures. Their interference is recorded by the photoelectron momentum detector. There is a one-by-one correspondence between the ionization time and the final momentum of the reference electrons. As a result, $\mathrm{PH}$ is a dynamic movie method encoding ultrafast electron dynamics with attosecond time resolution. The kinetic energy of signal electrons before rescattering can be as large as $3.17 U_{\mathrm{p}}[4]$, where $U_{\mathrm{p}}$ is the ponderomotive energy in the laser fields. The corresponding de Broglie wavelength could be comparable to or even smaller than the molecular internuclear distance $R$. Thus PH can achieve ångström spatial resolution.

Tunneling ionization (TI) is a fundamental process in strong-field sciences even though it is forbidden in classical physics. This instantaneous process, however, can hardly be studied since it occurs near the atomic and molecular cores.
PH provides us an indirect tool to probe TI. Zhou et al. [5] transfer the nonzero transverse displacement of the tunneling wavepacket from real to momentum space, thus enabling the tunneling exit to link to the phase change of the reference wave, which leads to the asymmetric PH pattern. Via mathematical models, they realized the accurate retrieval of the transverse tunneling exit by investigating the phase shift as a function of the transverse momentum. This work takes a step toward understanding the TI process, which will help reveal the mechanisms of other nonlinear phenomena, such as high-order harmonic generation and nonsequential double ionization. Moreover, as Zhou et al. [5] demonstrated, the tunneling wavepacket's displacement is closely related to the electron density distribution in molecules. Thus, PH has the potential to trace the attosecond charge migration in molecules, which is of essential importance for emerging attosecond chemistry.

1 D. Gabor, Nature 161, 777 (1948).

2 M. Spanner, O. Smirnova, P. B. Corkum, and M. Y. Ivanov, J. Phys. BAt. Mol. Opt. Phys. 37, L243 (2004).

3 Y. Huismans, A. Rouzée, A. Gijsbertsen, J. H. Jungmann, A. S. Smolkowska, P. S. W. M. Logman, F. Lépine, C. Cauchy, S. Zamith, T. Marchenko, J. M. Bakker, G. Berden, B. Redlich, A. F. G. van der Meer, H. G. Muller, W. Vermin, K. J. Schafer, M. Spanner, M. Y. Ivanov, O. Smirnova, D. Bauer, S. V. Popruzhenko, and M. J. J. Vrakking, Science 331, 61 (2011).

4 P. B. Corkum, Phys. Rev. Lett. 71, 1994 (1993).

5 Y. Zhou, J. Tan, M. Li, and P. Lu, Sci. China-Phys. Mech. Astron. 64, 273011 (2021).

*Corresponding author (email: xuebin.bian@wipm.ac.cn) 